\title{
Ethnic Group Attachment as The Main Predictor to Inculcate Altruistic Prosocial Behavior Among Malaysian Indigenous (Orang Asli) Adolescents
}

Aini Azeqa Ma'rof, Hanina H. Hamsan, Haslinda Abdullah \& Azlina Mohd Khir

To Link this Article: http://dx.doi.org/10.6007/IJARBSS/v11-i12/11798

DOI:10.6007/IJARBSS/v11-i12/11798

Received: 14 October 2021, Revised: 17 November 2021, Accepted: 30 November 2021

Published Online: 21 December 2021

In-Text Citation: (Ma'rof et al., 2021)

To Cite this Article: Ma'rof, A. A., Hamsan, H. H., Abdullah, H., \& Khir, A. M. (2021). Ethnic Group Attachment as The Main Predictor to Inculcate Altruistic Prosocial Behavior Among Malaysian Indigenous (Orang Asli) Adolescents. International Journal of Academic Research in Business and Social Sciences, 11(12), 552-565.

Copyright: (c) 2021 The Author(s)

Published by Human Resource Management Academic Research Society (www.hrmars.com)

This article is published under the Creative Commons Attribution (CC BY 4.0) license. Anyone may reproduce, distribute, translate and create derivative works of this article (for both commercial and non0-commercial purposes), subject to full attribution to the original publication and authors. The full terms of this license may be seen

at: http://creativecommons.org/licences/by/4.0/legalcode

Vol. 11, No. 12, 2021, Pg. 552 - 565

Full Terms \& Conditions of access and use can be found at http://hrmars.com/index.php/pages/detail/publication-ethics 


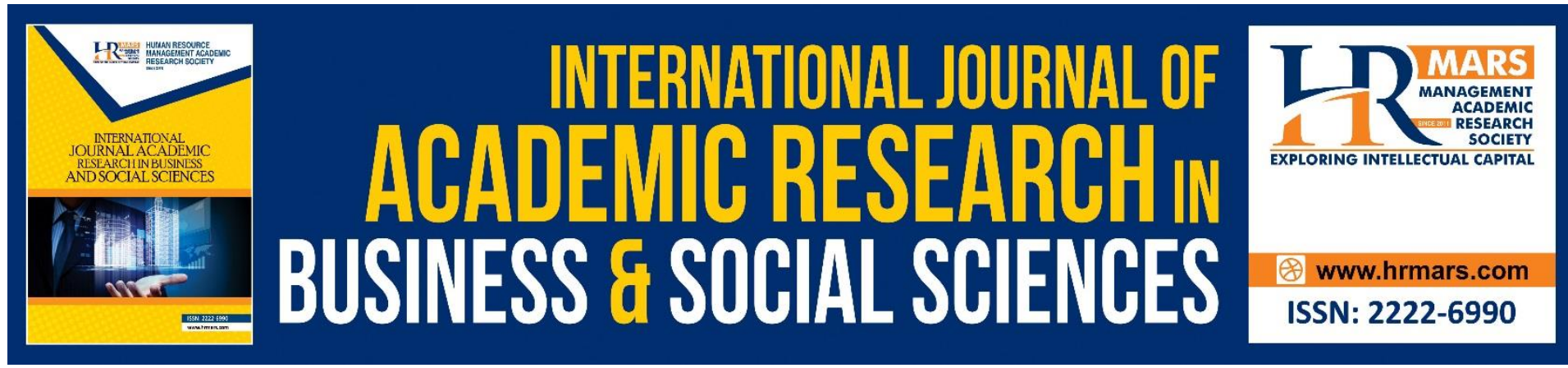

\title{
Ethnic Group Attachment as The Main Predictor to Inculcate Altruistic Prosocial Behavior Among Malaysian Indigenous (Orang Asli) Adolescents
}

\author{
Aini Azeqa Ma'rof, Hanina H. Hamsan, Haslinda Abdullah \& \\ Azlina Mohd Khir \\ Faculty of Human Ecology, Universiti Putra Malaysia, 43400 Serdang, Selangor Darul Ehsan, \\ Malaysia. \\ Email: azeqa@upm.edu.my
}

\begin{abstract}
Studies suggest that the Orang Asli adolescents who feel attached to their ethnic group engage in more altruistic prosocial behaviors. However, the psychological mechanisms that account for this association are not clear. Drawing on social identity and self-categorization theories, this study examined the association between social attachments (i.e., parental attachment, school attachment, peer attachment, and ethnic group attachment) and kindness value on altruistic prosocial behavior. Assessed among 402 Orang Asli adolescents, result shows that there were positive significant relationships between parental attachment, ethnic group attachment and kindness value on altruistic prosocial behavior. Meanwhile, there was a negative significant relationship of peer attachment on altruistic prosocial behavior. Further analysis revealed that ethnic group attachment served as the strongest predictor in inculcating altruistic traits among the Malaysian Orang Asli. The theoretical and practical implications of these findings were addressed.
\end{abstract}

Keywords: Orang Asli, Ethnic Group Attachment, Parental Attachment, Social Attachment, Altruistic Prosocial Behavior

\section{Introduction}

Prosocial behavior involves any action undertaken with the aim of benefiting others, regardless of one's underlying intention(s) (Piliavin et al., 1981). This behavior comprises any activity that is widely considered as beneficial from a societal standpoint, such as sharing, helping, volunteering, demonstrating social solidarity, and cooperating (Oberle et al., 2012). These phrases have been used interchangeably with prosocial behavior and have positive association with a variety of psychological processes that benefit both people and society (Eisenberg \& Mussen, 1989). However, the level to which people act prosocially varies significantly within and among cultures (Henrich et al., 2013). Thus, elucidating the roots of this variation may shed light on the mechanisms that promote altruistic prosocial intention particularly among the indigenous community. 
Indigenous people are well-known for their prosocial culture (Ma'rof \& Hamsan, 2020a). The Malaysian indigenous people who are called as the Orang Asli, is well-known with their cooperative culture, which means that any activity that benefits others is embedded in the Orang Asli's mind as a native group. Analytically, while individuals are more likely to assist members of their own group (in-group) than members of other groups (out-group), the close attachment between this community does not guarantee their mutual cohesion.

A collectivist-oriented society nurtures and nourishes prosocial values and behaviors, which benefit societal groups. Individualistic societies, on the other hand, put a priority on competitiveness and self-gain. The Orang Asli community, however, has been linked to other collectivist communities (Department of Orang Asli Affairs [JHEOA], 2008). Although majority of this community continues to live in a more rural, traditional, and patriarchal pattern of life, there is an emerging coexistence of traditional and modern features in the Malaysian indigenous society, which has the potential to alter their pattern of living and prosocial value. Meanwhile, an exploration of the significant associations between prosocial values and group attachments on altruistic prosocial behavior in Orang Asli adolescents remains unexplored. Hence, this study will delve deeper into this subject by investigating these domains.

Additionally, the study's focus is on the altruistic prosocial behavior of the Orang Asli adolescents. According to Ma'rof and Hamsan (2020b), there were among the Orang Asli adolescents particularly those who live in settlements near towns, have begun engaging in immoral behaviors such as smoking, skipping school, loitering, and motorcycle racing. Additionally, the Orang Asli are currently involved in drug misuse. As a result, it is vital to provide knowledge into their prosocial behavior in order to promote and encourage particularly their teenagers to act more prosocially and avoid engaging in any type of antisocial activity. Meanwhile, the Malaysian government's social development projects that focusing to this community, including educational support, were designed to expose this community to the outside world, diversity in contact, and attach to a larger community. JHEOA strives to establish an Orang Asli community that is responsive to the changes toward a sustainable balance of physical and mental growth through the initiatives brought about by the government. Thus, as a result of the government's efforts to develop this community and their exposure to the outside world, the possibility exists that the prosocial status of this community, which is well-known for its altruistic helping attitude, may change as a result of the exposure they encountered. Therefore, comprehending the social reality will result in a new understanding of the previously held prosocial conduct of this community.

\section{Prosocial Behavior among the Orang Asli People}

Culture as behavior, is a cumulative process and not a static entity. Thus, as is the case with other native minority communities in Malaysia, the survival and growth of the Orang Asli culture is inextricably related to their traditional culture, such as group members assisting one another (Ma'rof \& Hamsan, 2020a). Indigenous people are sometimes referred to as the Gemeinschaft community, which is defined as a small communion whose members share similar histories and life experiences, are familiar with one another, and engage in social interactions primarily among themselves.

The Orang Asli people sincerely cherish whatever values bond them together, value others, trust one another, promote harmony, and avoid conflict and quarrels. It is a way of reinforcing the Orang Asli unifying values. Additionally, by practicing sharing their crop produce, it 
became emblematic of their strong friendship. There is no reciprocal or egoistic nature of assistance involves, whereby the prosocial acts underlie solely by altruistic act (Ma'rof \& Hamsan, 2020b). All beneficial actions are based on sincerity and togetherness. However, the exposure of Orang Asli to the mainstream community may have an indirect effect on the prosocial behavior of this marginalized community. Therefore, this study will examine if their attachment to a particular social context will persist or influence their altruistic behavior.

\section{Social Attachments and Prosocial Behavior}

Attachment which defined as a persistent psychological connection between human beings, played a critical part in the development of prosocial behaviors. According to Attachment Theory (Bowlby, 1980), adolescents who have strong emotional connections with their significant others contribute to the development of a stable attachment style that benefits the adolescent's socio-emotional and physical well-being. Adolescents' helping behavior may be influenced by social systems such as families (Nie et al., 2016), schools (Caprara et al., 2014), peers (Schoeps et al., 2020), and ethnic group (Arment et al., 2011). It is therefore crucial to ascertain the social attachment factors that influence adolescent altruism, specifically among Orang Asli adolescents.

\section{Parental and Ethnic Group Attachments}

The socialization between parent and their child frequently serves as a lifelong relationship. Parents serve as the key socialization agents for their children, particularly in terms of moral judgement and prosocial behavior (Padilla-Walker, 2014). Attachment, specifically parental attachment, or the stable, loving, and warm relationship between parent and child, has been positively associated with prosocial behavior in adolescents (Richaud et al., 2013). Additionally, numerous theories and empirical evidence support the widely held belief that parents play a critical role in promoting and fostering prosocial conduct in adolescents (Grusec et al., 2000). However, there are still limited studies has proven this from the context of the indigenous people.

Meanwhile, from the context of ethnic group attachment, Sidanius et al (1997) defines it as the psychological identification with ethnic groups or heritage. This implies that ethnic group serves as the foundation for the development of individual self-perception. Ethnic attachment is also a social process that influences how friends are chosen, their life perspectives, reactions, and social reactions that are accepted in the social context. While an individual may mentally identify with an ethnic group, the power and authenticity of such identity are dependent on the acceptance and acknowledgment of members of the group.

Group attachment and group identity are inextricably linked. A person is said to have a strong ethnic group attachment if they continue to practice and retain their ethnic identity; thus, ethnic identity is the primary factor and is inextricably linked to determining a person's ethnic group attachment. Nevertheless, Sanders (2002) asserts that ethnic boundaries define a group, rather than being described from an ethnicity's cultural perspective. However, in Malaysia, ethnicity and culture are inextricably linked because an individual can be defined through physical and cultural characteristics observed in daily life.

Adding to this, Armenta et al (2010) conducted a study on ethnic group attachment and prosocial behavior based on the Social Identity theory on the American-Mexican community. 
This study revealed that individuals with strong ethnic group attachment exhibited altruistic acts motivated by strong family values. While values from the dominant culture (in this case, American values) results in egoistic prosocial behavior, that is, helpful action that is based on reciprocity. This assistance in the form of egoistic prosocial activity is provided in order to seek for future mutual help. However, it is still unclear whether from the exposure of the Orang Asli to the mainstream community will influence their altruistic prosocial act that yet to be explored.

\section{Peer and School Attachments}

Individuals' ability to form and sustain meaningful peer relationships is considered essential for a healthy socio-emotional adjustment across the lifespan. Padilla-Walker and Carlo (2014) argue that peer relationships appear to have a substantial impact in teenagers' prosocial development. According to a study conducted by Laible et al (2000), attachment security with peers was associated with a high level of empathy, demonstrating how supportive relationships with peers can bolster adolescents' concern and reaffirm their responsibility toward others. Additionally, the secure attachment with peers can have a profound effect on an individual's prosocial behavior and engagement within society and community.

According to Tomé et al (2012), peer influence develops during adolescence, while family interaction declines. Adolescents place a high value on friendships and cliques as a social setting. Thus, it is obvious that the peer group is a significant agent of achievement, ritualistic, and socialization (Ryan, 2000). Adolescents' attachment to school and friends provides them with a variety of exposure and choices to consider and act on. Additionally, they have an effect on adolescents' responses to their evaluations via retention or changes in motivation and participation in an activity (Vagos \& Carvalhais, 2020). As a result, peer attachment may also function as a training ground for anti-social behaviors and misdeeds (Murphy et al., 2017).

Meanwhile, from the school context, school is a primary setting for social interaction, personal skill development, peer group formation, self-expression, and self-improvement (Carolan \& Chesky, 2012). Numerous studies have demonstrated that the social context of the school plays a significant role in deciding whether or not an adolescent will adopt a prosocial path during adolescence. Carlo et al. (1999) found that environmental transitions and certain components of schooling had a significant effect on students' prosocial and moral conduct. Additionally, schools that foster values of connectedness and cooperation in their environment may have an influence on adolescent involvement in prosocial activities (Eisenberg et al., 2006).

Undoubtedly, the Orang Asli are the descendants of Peninsular Malaysia's early inhabitants. It has been asserted that they retained a great deal of their identity and values to the present day because of their relative isolation from other communities and development. This is not to suggest that the Orang Asli lived in utter isolation, since several programmes by the Malaysian government have been implemented to enhance the Orang Asli community's standard of living, such as encouraging the Orang Asli children to attend school. This indirectly exposes them to the development and enables them to mingle and socialize with members of different races. Therefore, this study will examine the altruistic prosocial behavior of this 
group of adolescents in regard to their attachment with various forms of social groups and prosocial value.

\section{Method}

\section{Participants}

Participants comprised of 402 Orang Asli adolescents from 10 public secondary schools in the Malaysian states of Perak and Pahang. Both states were chosen as majority of the Orang Asli people resided in these states. Females ( $n=245,60.90 \%$ ) and males ( $n=157,39.10 \%$ ) between the ages of $14(n=232,57.72 \%)$ and $16(n=170,42.28 \%)$ years old participated in this study. Majority of the respondents (96.76\%) were from the Senoi tribe, followed by $2.24 \%$ from Proto-Malays tribe, and $1 \%$ was from the Negritos tribe. The Senoi tribe is concentrated in the Malaysian states of Perak and Pahang, which explains majority of the respondents came from this tribe (sub-ethnic). Meanwhile, since this study was conducted in a school setting, thus majority of the respondents (77.1\%) stayed in JAKOA-provided school hostels due to their settlements' remoteness and proximity to schools, while the remaining $22.9 \%$ of respondents lived with their families, particularly those in the Cameron Highlands, Pahang area.

\section{Data Collection}

A cross-sectional design method was used to employ a quantitative approach via a selfadministered questionnaire. Students' names were obtained from school counsellors and classroom teachers' weeks before to data collection for each of the ten secondary schools in Perak and Pahang. Students' samples were drawn from the list using systematic random sampling. Students were gathered in a classroom or the school hall for data collection. A selfadministered questionnaire is utilized because it is more appropriate as respondents are free to complete the task without the researcher's "interference." However, there were some students that have limited proficiency in Malay language and do not master basic literacy skills, therefore were unable to read and comprehend the task. Thus, with the assistance of a research assistant, the researcher gathered these students in a group for one-on-one assistance by reading and clarifying each item.

\section{Measures}

Altruistic prosocial behaviour. Altruistic prosocial behaviour was measured by using the Prosocial Tendencies Measure-Revised (PTM-R) by Carlo and Randall (2002). The measurement consists of six subscales; altruistic, compliant, emotional, public, anonymous and dire. However, for this study; only the altruistic subscale was used which consists of altruistic and anonymous prosocial behaviours that represents the altruistic prosocial behaviour by definition. Examples of the items are "I think one of the best things about helping others is that it makes me look good" (altruistic), and "I think that helping others without them knowing is the best type of situation" (anonymous). Respondents were asked to rate to the statement on a four-point scale ranging from 1 (totally disagree) to 4 (totally agree). The reliability was $\alpha=.76$.

Parental attachment. Attachment to parents was measured using the Parental Attachment Questionnaire (PAQ) by Kenny, Griffiths, and Grossman (2005). The PAQ contains three scales: Affective Quality of Attachment, Parental Fostering of Autonomy, and Parental Role in Providing Emotional Support. However, for the purpose of this study, 7 items were selected 
to assess the Affective Quality of Attachment. Examples of items includes, "In general, my parents understand my problems and concern", and "During time spent together, my parents were persons for whom I felt a feeling of love" with indicators of 1 (Strongly agree) to 4 (Strongly disagree). The reliability was $\alpha=.78$.

Peer attachment. Inventory of Parent and Peer Attachment (IPPA, Armsden \& Greenberg, 1987) was used to assess peer attachment. The IPPA was developed to assess adolescents' perceptions of the positive and negative affective/cognitive dimensions or relationships with parents and close friends. 8 items from the inventory that particularly assess the students' attachment with their peer was used with a reliability of $\alpha=.79$. Examples of items are "My friends can tell when I'm upset about something", I like to get my friends' point of view on things I'm concerned about" with indicators of 1 (Strongly agree) and 4 (Strongly disagree).

School attachment. School connectedness Scale (SCS) by Furlong, O’Brennan, and You (2011) was used to measure adolescents' attachment to school environment. This scale is designed to measure the bond students' feel toward school and, as such, measures psychological and non-academic, behavioural, and cognitive engagement. Examples of questions are "I feel proud to be in this school", I feel save and comfort in school", "I feel attached with people in my school, "Teachers in my school treat students fairly" with the response scales of 1 (Strongly disagree) to 4 (Strongly agree). The reliability is $\alpha=.78$.

Ethnic group attachment. Ethnic group attachment was measured using the Multigroup Measure of Ethnic Identity (MEIM; Phinney, 1992). MEIM consists of 18 items assessing three aspects of ethnic identity: positive ethnic attitudes and sense of belonging, ethnic identity achievement, and ethnic behaviours or practices. Items are rated on a four-point scale from 1 (strongly disagree) to 4 (strongly agree). Examples of items includes "I am happy that I am a member of the group I belong to", "I have spent time trying to find more about my ethnic group, such as its history, traditions, and customs", "I participate in cultural practices of my own group, such as special food, music, or customs". The reliability was $\alpha=.77$.

Kindness. Values-in-action Inventory of Strengths for youth (VIA-youth; Peterson \& Seligman, 2004) was used to assess the extent to which adolescents valued and enjoyed helping and being kind to others. The VIA-youth assess 24 different values (e.g., bravery, creativity). As for the present study, 10 items from the kindness subscale were adopted to examine participants prosocial value. Respondents rated statements based on a four-point scale with 1 (strongly agree) to 4 (strongly agree). The reliability was $\alpha=.84$.

The questionnaire items were translated from English to Malay and then back to English using repeated translation (Rich et al., 1996). This procedure is used to ensure the accuracy of translated items. Due to the targeted respondents' limited fluency in Bahasa Malaysia, experts in Orang Asli research were chosen to examine the items and ensure that the language used in the items is understood by them (the Malay language).

\section{Results and Discussion}

The association between social attachments, kindness value and altruistic prosocial behavior is illustrated in Table 1 . The results indicate that parental attachment $(r=.185, p<$ $.001)$, ethnic group attachment $(r=.305, p<.001)$ and kindness value $(r=.262, p<.001)$, have a positive significant correlation with altruistic prosocial behavior. Meanwhile, a negative 
association between altruistic prosocial behavior and peer attachment was revealed $(r=-$ $.153, p<.001)$ with non-significant relationship identified on school attachment. These findings imply that teenagers who are more devoted to their parents and ethnic group together embrace the kindness value are more likely to engage in altruistic prosocial behavior.

Social settings and ethnic group are two elements that can determine whether adolescents value prosocial behavior. Additionally, these findings corroborate the relations concept based on the Social Exchange Theory (Homans, 1974), under which in a collectivist society as practiced by the Orang Asli, the existence of communal relationships within their community and with significant others (e.g., parents and ethnic group) fosters an appreciation for kindness in comparison to their attachment to the outside community (e.g., peer). Additionally, ethnic group attachment can advocate for adolescents' positive attitudes and prosocial values. Thus, the Orang Asli community, which values sharing, peace, and village cohesion, may instill a sense of kindness in their adolescents (Endicott \& Dentan, 2004).

Meanwhile, a healthy attachment between parent and child promotes the development of competence and social skills, as well as empathy and understanding of another's position, all of which have been shown to promote prosocial values. In the context of the Orang Asli, many Orang Asli families cherish familial interdependence and have been shown to be less conflictual than other Malaysian mainstream cultures (Endicott \& Dentan, 2004). A close and loving interaction between parent and child is likely to instill favorable prosocial values in Orang Asli adolescents. It has been observed that Orang Asli families exhibit unique features, such as family dependency. This includes, but is not limited to, the flexible assignment of duties among family members and reliance on extended relatives or support.

Another social setting is peer attachment. The reason for the declining of altruistic prosocial behavior in the Orang Asli adolescents could be due to the poor treatment they experienced in school and among peers (as for example taunting and bullying). Additionally, another aspect that may contribute to the decrease in the kindness value of Orang Asli children is their attachment to peers, as they are uninterested in the school curriculum and others are tired of being reprimanded by teachers. Additionally, it is alleged that some youngsters would prefer to assist their parents at work or were uncomfortable to attend school. Given that Orang Asli are a group that is often deficient in motivation and self-esteem (Hussain et al., 2020), the circumstances they confront at school and in their relationships with peers may cause them to lose their sense of kindness toward others. Orang Asli have a strong peaceful image of themselves and self-identify as nonviolent people not easily get angry or would strike others (Endicott \& Dentan, 2004). A self-image that encourages aggressiveness contradicts their definition of virtue, resulting in a decline in prosocial value when in school or engaging with peers. 
Table 1 The relationship between social attachments and prosocial value (kindness).

\begin{tabular}{lcc}
\hline Variable & \multicolumn{2}{c}{ Altruistic prosocial behavior } \\
\cline { 2 - 3 } & $\boldsymbol{r}$ & $\boldsymbol{p}$ \\
\hline Social Attachments & & \\
Parental attachment & $.185^{* *}$ & .000 \\
Peer attachment & $-.153^{* *}$ & .002 \\
School attachment & -.066 & .184 \\
Ethnic group attachment & $.305^{* *}$ & .000 \\
Prosocial value & & \\
Kindness & & .000 \\
\hline
\end{tabular}

Note: ${ }^{* * *}$ Level of significant is at $p<0.001$

Table 2 reported the main indicator that contribute to altruistic prosocial behavior. Overall, the model shows a significant with $\mathrm{R}^{2}=.24$. This shows that this model contributes $24 \%$ variance to altruistic prosocial behaviour with $F=13.27, p<.001$. Meanwhile, only ethnic group and kindness contributed significantly towards altruistic prosocial behaviour with ethnic group attachment $(B=.20, p<.000)$ shows the strongest predictor to altruistic prosocial behaviour followed by kindness $(B=.19, \mathrm{p}<.000)$.

In terms of ethnic group attachment, Orang Asli, as a collective culture that nurture connectedness and in-group needs, are more inclined to help in-group but less likely to help out-group members than people from individualistic cultures (Aronson et al., 2004). This community has developed an inclination for in-group cohesion, which may foster a strong attachment to their groups and hence promote altruistic prosocial behaviour. Additionally, Triandis et al., (1988) in their studies indicated that collectivists place a higher value on integrity and have an intense emotional bond with their in-group. As a result, this study provides insights on the role of ethnic group attachment in cultivating altruistic behaviour among the Orang Asli community.

Other-oriented effects (sympathy) or other internalized principles such as kindness may drive altruistic prosocial behavior (Carlo \& Randall, 2002). Thus, altruism is usually motivated by internalized prosocial goals and is positively connected with internalized moral reasoning. As the Orang Asli valued kindness as a source of unity, it has been fostered throughout the community as a fundamental aspect in encouraging prosocial behavior. 
Table 2 Multiple regression in determining the main indicators of online pornography consumption on the tendencies towards IPD.

\begin{tabular}{lcccc}
\hline Variable & \multicolumn{4}{c}{ Altruistic Prosocial Behaviour } \\
\cline { 2 - 5 } & B & SE. B & Beta, $\boldsymbol{\beta}$ & $\boldsymbol{p}$ \\
\hline Social Attachments & & & & \\
Parental attachment & .06 & .06 & .05 & .087 \\
Peer attachment & -.02 & .05 & -.01 & .121 \\
Ethnic attachment & .12 & .03 & .20 & .000 \\
& & & & \\
Prosocial Value & & & & .000 \\
Kindness & .15 & .04 & .19 & \\
\hline Adjusted $\mathbf{R}^{\mathbf{2}}$ & .24 & & & \\
F & 13.27 & & & \\
\hline
\end{tabular}

\section{Theoretical and Policy Implications}

The Orang Asli are not actively or directly participating in the mainstream economy and appear to uphold behaviors and attitudes that promote group cohesiveness and cooperation. Thus, for Indigenous communities that have a strong connection to their ethnic heritage, the proclivity for altruistic behavior remains high. From this study, the Orang Asli adolescents demonstrated high level of ethnic attachment when compared to other social attachments. This shows that the Orang Asli's reliance on their ethnic group throughout the school year and their return to their respective villages only during school breaks (once every three to six months), but this does not weaken their ethnic identity.

As a result of this study, it is clear that ethnic group attachment and kindness value have an effect on prosocial behavior in the Orang Asli collectivist culture. Harmony and interdependence among group members are emphasized and cherished in the Orang Asli culture. Group members are relatively close psychologically and emotionally, which fosters a strong tie between them. Members of this culture are expected to behave prosocially, which is learnt through socialization.

Overall, the findings indicate that the altruistic prosocial behavior in the Orang Asli community, particularly among their school-aged adolescents, has remained stable. On the other hand, respondents' socialization with the external community (e.g., peers and school) altered their intention to help others, which is underpinned by egoistic conduct (Ma'rof \& Hamsan, 2020a). The findings revealed that the Orang Asli adolescents retain significant ties to their family and ethnic group, which encourages them to help others, particularly those in their own community. These findings supported Bowlby's (1980) Attachment Theory, which asserts that adolescents who feel secure and connected to their in-groups, such as their parental and ethnic communities, exhibit greater proclivity for altruistic behavior. Commitment and participation with the group result in the prosocial act.

Meanwhile, according to Social Exchange Theory (Homans, 1974), before a person assists others, they examine the costs and benefits of the help and their relationship with the beneficiaries. According to the study, Orang Asli adolescents who are strongly attached to their parents and ethnic group exhibit altruistic prosocial conduct, which is consistent with communal relationships in which people feel a personal responsibility to assist those in need. 
Additionally, another study by Ma'rof and Hamsan (2020a) found that interactions with peers at school exhibit egoistic prosocial behavior. This therefore reinforces the exchange relationships in which help is provided in exchange for bearing costs and obtaining benefits. In conclusion, this study contributes to the field of prosocial behavior by examining it from an indigenous perspective.

\section{Policy Implications and Recommendations}

This study's findings have ramifications for parents, psychologists, educators, and policymakers. One clear implication for parents is that increased parental warmth and attachment, as measured by parents' use of positive and bidirectional communication, reasoning practice, and emotional involvement, are beneficial for the development of adolescents' altruistic prosocial behavior. The same also implies to their ethnic community. The findings will assist parents in recognizing the critical role of their engagement in boosting their child's prosocial behavior and in making subsequent adjustments to improve the quality of their parenting and community behavior.

Additionally, there is a need to make a concerted effort to promote prosocial behavior and secure attachment among teenagers, not just in their immediate community but also in the larger community (e.g., the school and mainstream community). This also includes the government's role, specifically the Department of Orang Asli Development, as well as the role of non-governmental organizations (NGOs) such as the Center for Orang Asli Concern (COAC), Peninsular Indigenous Village Network (JKOASM), and the Malaysian Orang Asli Federation (YOAM) in promoting prosocial behavior among the Orang Asli community, particularly their youth. This is crucial as it fosters adolescents' sense of belonging to the larger community, which in turn encourages their prosocial behavior, particularly in caring for those beyond their community circle.

Additionally, the findings of this study shed light on the critical function of ethnic group attachments in teenagers' development of altruistic prosocial behavior. This demonstrates that the communities are capable of instilling prosocial conduct and values in their children. Adolescents' altruistic prosocial behavior was indirectly influenced by ethnic group attachments via the kindness value they adhere to. However, the present study could not demonstrate that parental and ethnic group attachments are more effective at fostering prosocial behavior than peer and school attachments. Thus, additional research is required to corroborate these findings. Moreover, this study revealed that the ecosystem variables such as cultural values play a substantial role in adolescent attachment to certain groups and, consequently, in adolescents' altruistic prosocial behavior. Therefore, this study suggests that a certain attachment component (i.e., acceptance and warm treatment from the parent, ethnic group, and society) had a beneficial effect on the development of teenagers' altruistic prosocial behavior.

Overall, it is reassuring to find that prosocial behavior can be effectively fostered and developed. Understanding that positive features of human functioning are influenced by external factors and that we can act appropriately to prevent violence and aggression by supporting the value of kindness and compassion enables us to transform social science into effective social policy and practice. 


\section{Conclusion}

The transition of the Orang Asli people is evident in all spheres of their lives, including social, economic, educational, and cultural. Indeed, society's enlightenment is critical as a catalyst for mental modernization towards development and a higher standard of living. Government strategies aimed at integrating Orang Asli can be classified into two categories: 1) mainstreaming these groups; and 2) assimilate into the Malay community to bring them together in line with the mainstream progress. Both policies shape the Orang Asli identity and will have an indirect effect on their prosocial beliefs. Along with the government's efforts to mainstream the Orang Asli, it is vital to consider the measures that can be made to preserve the Orang Asli's identity and culture. The influence of modernization, which introduces new cultures and identities, will dilute not just the Orang Asli's identity and rituals, but also those of mainstream society. It should be noted that not all development and change are beneficial, particularly in terms of preserving the fundamental values and foundations of community life.

\section{Reference}

Armsden, G. C., \& Greenberg, M. T. (1987). The inventory of parent and peer attachment: Individual differences and their relationship to psychological well-being in adolescence. Journal of youth and adolescence, 16(5), 427-454.

Bowlby, J. (1980). Attachment and loss: Volume III: Loss, sadness and depression. In Attachment and Loss: Volume III: Loss, Sadness and Depression (pp. 1-462). London: The Hogarth press and the institute of psycho-analysis.

Caprara, G. V., Kanacri, B. P. L., Gerbino, M., Zuffiano, A., Alessandri, G., Vecchio, G., ... \& Bridglall, B. (2014). Positive effects of promoting prosocial behavior in early adolescence: Evidence from a school-based intervention. International Journal of Behavioral Development, 38(4), 386-396.

Carlo, G., \& Randall, B. A. (2002). The development of a measure of prosocial behaviors for late adolescents. Journal of

Carlo, G., \& Randall, B. A. (2002). The development of a measure of prosocial behaviors for late adolescents. Journal of

Carlo, G., \& Randall, B. A. (2002). The development of a measure of prosocial behaviors for late adolescents. Journal of

Carlo, G., \& Randall, B. A. (2002). The development of a measure of prosocial behaviours for late adolescents. Journal of Youth and Adolescence, 20, 334-358.

Carlo, G., Crockett, L. J., Randall, B. A., \& Roesch, S. C. (2007). A latent growth curve analysis of prosocial behavior among rural adolescents. Journal of Research on Adolescence, 17(2), 301-324.

Carolan, B. V., \& Chesky, N. Z. (2012). The relationship among grade configuration, school attachment, and achievement: Focusing on middle grades students' sense of school attachment may be more effective than reorganizing school grade configuration. Middle School Journal, 43(4), 32-39.

Department of Orang Asli Affairs. (2008). Annual report of Department of Orang Asli Affairs 2008. Kuala Lumpur: Jabatan Hal Ehwal Orang Asli.

Eisenberg, N., \& Mussen, P. H. (1989). The roots of prosocial behavior in children. Cambridge University Press.

Eisenberg, N., Spinrad, T. L., \& Sadovsky, A. (2006). Empathy-related responding in children in Handbook of Moral Development. New York and London Psychology Press. 
Endicott, K., \& Dentan, R. K. (2004). Into the mainstream or into the backwater. Civilizing the margins: Southeast Asian government policies for the development of minorities, 2455.

Furlong, M. J., O'Brennan, L. M., \& You S. (2011). Psychometric properties of the ADD Health School Connectedness Scale for 18 sociocultural groups. Psychology in the Schools, 48(10), 986-997.

Grusec, J. E., Goodnow, J. J., \& Kuczynski, L. (2000). New directions in analyses of parenting contributions to children's acquisition of values. Child development, 71(1), 205-211.

Homans, G. C. (1974). Social behavior and its elementary forms. New York: Harcourt, Brace and World.

House, B. R., Silk, J. B., Henrich, J., Barrett, H. C., Scelza, B. A., Boyette, A. H., ... \& Laurence, S. (2013). Ontogeny of prosocial behavior across diverse societies. Proceedings of the National Academy of Sciences, 110(36), 14586-14591.

Kenny, M. E., Griffiths, J., \& Grossman, J. (2005). Self-image and parental attachment among late adolescents in Belize. Journal of adolescence, 28(5), 649-664.

Laible, D. J., Carlo, G., \& Raffaelli, M. (2000). The differential relations of parent and peer attachment to adolescent adjustment. Journal of Youth and adolescence, 29(1), 45-59.

Layous, K., Nelson, S. K., Oberle, E., Schonert-Reichl, K. A., \& Lyubomirsky, S. (2012). Kindness counts: Prompting prosocial behavior in preadolescents boosts peer acceptance and well-being. PloS one, 7(12), e51380.

Ma'rof, A. A., \& Hamsan, H. H. (2020a). Altruistic and egoistic prosocial behaviors among Orang Asli adolescents: Its relations to attachments and prosocial value. International Journal of Academic Research in Business and Social Sciences, 10(16), 58-72.

Ma'rof, A. A., \& Hamsan, H. H. (2020b). Altruistic Prosocial Behavior and Attachments among Orang Asli Adolescents: The Mediating Role of Prosocial Value. International Journal of Academic Research in Business and Social Sciences, 10(16), 401-411.

Murphy, T. P., Laible, D., \& Augustine, M. (2017). The influences of parent and peer attachment on bullying. Journal of child and family studies, 26(5), 1388-1397.

Nie, Y. G., Li, J. B., \& Vazsonyi, A. T. (2016). Self-control mediates the associations between parental attachment and prosocial behavior among Chinese adolescents. Personality and Individual Differences, 96, 36-39.

Padilla-Walker, L. M. (2014). Parental socialization of prosocial behavior: A multidimensional approach. Oxford University Press.

Peterson, C., \& Seligman, M. E. P. (2004). Character strengths and virtues: $A$ handbook and classification. American Psychological Association; Oxford University Press.

Phinney, J. (1992). The Multigroup Ethnic Identity Measure: A new scale for use with adolescents and young adults from diverse groups. Journal of Adolescent Research, 7, 156-176.

Piliavin, J, M., Dovidio, J. F., Gaertner, \& Clark, R, D. (1981). Emergency Intervention, New York: Academic Press.

Richaud, M. C., Mesurado, B., \& Lemos, V. (2013). Links between perception of parental actions and prosocial behavior in early adolescence. Journal of Child and Family Studies, 22(5), 637-646.

Ryan, A. M. (2000). Peer groups as a context for the socialization of adolescents' motivation, engagement, and achievement in school. Educational psychologist, 35(2), 101-111.

Sanders (2002). Ethnic Boundaries and Identity in Plural Societies. Annual Review of Sociology, 28, 327-357. 
Schoeps, K., Mónaco, E., Cotolí, A., \& Montoya-Castilla, I. (2020). The impact of peer attachment on prosocial behavior, emotional difficulties and conduct problems in adolescence: The mediating role of empathy. PloS one, 15(1), e0227627.

Sidanius, J., Feshbach, S., Levin, S., \& Pratto, F. (1997). The Interface Between Ethnic and National Attachment: Ethnic Pluralism or Ethnic Dominance? The Public Opinion Quarterly, 61(1), 102-133.

Hussain, S. T. P. R., Krishnasamy, D. S., \& Hassan, G. A. A. (2020). Resettlement of the Orang Asli and development plan for Orang Asli community in Malaysia. Journal of TechnoSocial, 9, 32-43.

Tomé, G., Matos, M., Simões, C., Diniz, J. A., \& Camacho, I. (2012). How can peer group influence the behavior of adolescents: explanatory model. Global journal of health science, 4(2), 26-35.

Triandis, H. C., Bontempo, R., Villareal, M. J., Asai, M., \& Lucca, N. (1988). Individualism and collectivism: Cross-cultural perspectives on self-ingroup relationships. Journal of Personality and Social Psychology, 54(2), 323-338.

Vagos, P., \& Carvalhais, L. (2020). The impact of adolescents' attachment to peers and parents on aggressive and prosocial behavior: A short-term longitudinal study. Frontiers in Psychology, 11. 